\title{
Y tú, ¿te proteges del sol? Un proyecto STEM con mirada científica
}

\author{
Fina Guitart \\ CESIRE - Àmbit cientific. Centre de Recursos Pedagògics Específics de Suport a la Innovació i a la Recerca \\ Educativa. Barcelona.España.jguitar3@xtec.cat \\ ORCID: bttps:/ / orcid.org/0000-0002-0170-9068 \\ Silvia Lope \\ CESIRE - Àmbit cientific. Centre de Recursos Pedagògics Específics de Suport a la Innovació i a la Recerca \\ Educativa. Barcelona. España.slope@xtec.cat \\ ORCID: https://orcid.org/0000-0003-3192-1059
}

[Recibido: 6 Febrero 2019. Revisado: 25 Abril 2019. Aceptado: 15 Julio 2019]

\begin{abstract}
Resumen: Se presentan un conjunto de actividades que pueden ser utilizadas como embrión para construir un proyecto de aprendizaje que puede desarrollarse en los niveles de $3^{\circ}$ o $4^{\circ}$ de la ESO y, con algunas adaptaciones, también en primero de bachillerato. Se propone el reto de encontrar respuesta a preguntas relevantes en torno a contenidos científicos relacionados con la radiación solar y las repercusiones de ésta en la propia salud y en la de la población. La indagación, el diseño y realización de experimentos, así como el registro de datos y su interpretación tienen una especial relevancia a lo largo de toda la propuesta. En las diferentes actividades se indican las relaciones entre las ideas que se trabajan y el modelo científico concreto al que corresponden, proporcionando así una ocasión para contribuir a la construcción de estos modelos. Finalmente se reflexiona sobre el funcionamiento de la propuesta cuando se ha trabajado con alumnos de ESO y cuando se ha presentado en cursos de profesorado.
\end{abstract}

Palabras clave: Radiación solar; UVA; UVB; Experimentación; Modelización; Proyectos.

And you, do you protect yourself from the sun? A STEM project with a scientific perspective

Abstract: We present a set of activities that can be used as a starting point to build a learning project that can be developed in the 3rd or 4th levels of ESO and, with some adaptations, also in the first year of high school. The challenge is to find answers to relevant questions related to scientific contents related to solar radiation and the repercussions of this on one's own health and that of the population. The investigation, the design and execution of experiments as well as the data recording and its interpretation have a special relevance throughout the whole proposal. In the different activities, the relationships between the ideas that are worked on and the concrete scientific model to which they correspond are indicated, thus providing an opportunity to contribute to the construction of these models. Finally, it reflects on the operation of the proposal when it has been implemented with ESO students and when it has been presented in teacher training courses.

Keywords: Rolar radiation; UVA; UVB; Experimentation; Modeling; Project.

Para citar este artículo: Guitart. F. y Lope. S. (2019) Y tú, ¿te proteges del sol? Un proyecto STEM con mirada científica. Revista Eureka sobre Enseñanza y Divulgación de las Ciencias 16(3), 3202. doi: 10.25267/Rev_Eureka_ensen_divulg_cienc.2019.v16.i3.3202

\section{Introducción}

El concepto de educación STEM (acrónimo de Science, Technology, Engineering, Mathematics) propone involucrar al alumnado en procesos de investigación científica para el aprendizaje, de forma más o menos integrada, de nuevos conceptos de ciencias, tecnología y matemáticas en el seno de un proceso práctico de diseño y resolución de problemas, de manera similar a como se hace en el mundo real (Fortus et al 2005). Si bien los inicios del concepto STEM están ligados a la necesidad de despertar más vocaciones científico tecnológicas: "Para mantenerse

\author{
Revista Eurek a sobre Enseñanza y Divulgación de las Ciencias \\ Universidad de Cádiz. APAC-Eureka. ISSN: 1697-011X \\ http:/ / dx.doi.org/10.25267/Rev_Eureka_ensen_divulg_cienc.2019.v16.i3.3202 \\ http:/ / reuredc.uca.es
}


competitivos en una economía global en crecimiento es imperativo que mejoremos los resultados de los alumnos en las asignaturas STEM" (Wang, Moore, Roehrig, Park 2011), también es cierto que superar los retos relacionados con aspectos socio científicos que tiene la sociedad actual requiere que todos los ciudadanos tengan una mejor comprensión de la ciencia y de la tecnología, para que puedan participar de forma activa y responsable en las decisiones sobre dichos temas (Hazelkorn, Charly, Yves, Constantinos, Ligia, Michel, Welzel-Breuer 2015).

La investigación actual de la aplicación del Aprendizaje Basado en Proyectos (ABP) y Educación STEM demuestra que la realización de Proyectos puede aumentar el interés de los alumnos en Ciencias, Tecnología, Ingeniería, y Matemáticas (STEM), ya que involucran a los estudiantes en la solución de problemas auténticos, trabajan en equipo y construyen soluciones reales y tangibles (Fortus et al 2005).

Sin embargo, en la actualidad el profesorado dispone aún de pocos ejemplos publicados de proyectos STEM y este hecho proporciona una cierta inseguridad sobre si lo que está haciendo en el aula se ajusta o no a un buen proyecto. Además, con frecuencia se invoca el mito que los proyectos STEM implican necesariamente construir algún artefacto $\mathrm{y}$, preferentemente, utilizando tecnologías creativas sofisticadas. En este sentido hemos querido compartir una experiencia que consideremos STEM, con mirada científica, que involucra, a partir de un contexto auténtico, la construcción de contenidos y habilidades científicas relevantes, a la vez que hace necesario comprender e interiorizar el funcionamiento de herramientas tecnológicas, que propone actividades de trabajo en equipo y, todo ello, orientado a la acción y la toma de decisiones sobre la propia salud y la de la población.

La propuesta que se presenta a continuación es un conjunto de actividades que pueden ser utilizadas para construir un proyecto de aprendizaje que puede desarrollarse en los niveles de $3^{\circ}$ o $4^{\circ}$ de la ESO y, con algunas adaptaciones, también en primero de bachillerato. Se trata de una propuesta abierta y, dependiendo de los intereses del alumnado o de la intencionalidad del profesorado, se pueden ir añadiendo actividades o eliminar alguna.

\section{Punto de partida}

La idea es partir de una situación habitual en la vida de los alumnos (¿'Te proteges del Sol?), se propone de la lectura de un pequeño texto, un dialogo entre adolescentes en el que hablan sobre sus dudas sobre los riesgos-beneficios de tomar el sol y sus hábitos de protección. La finalidad de esta actividad es compartir los objetivos de aprendizaje con el alumnado, explorar sus idees previas, así como despertar su interés sobre los posibles efectos de la exposición a la radiación solar y los hábitos de salud relacionados: ¿Son realmente conscientes de los riesgos que puede tener el exceso de sol? ¿Conocen las causas de estos riesgos? ¿Saben cómo prevenirlos o minimizarlos? ¿Y qué saben sobre los beneficios? ¿Es importante tomar el sol moderadamente?

Será importante generar buenas preguntas que orienten las siguientes actividades. Se propone animar a los alumnos a formular preguntas relacionadas con el tema y, posteriormente, priorizarlas (elegir las de más interés de manera consensuada) y agruparlas identificando aquellas que se podrán resolver buscando en diferentes fuentes fiables de información y aquellas que podrán ser investigadas experimentalmente. La ordenación de estas preguntas proporcionará el hilo conductor del proyecto.

Nuestra propuesta se organiza en torno a tres ejes:

- ¿Qué contiene la radiación que nos llega del Sol? 


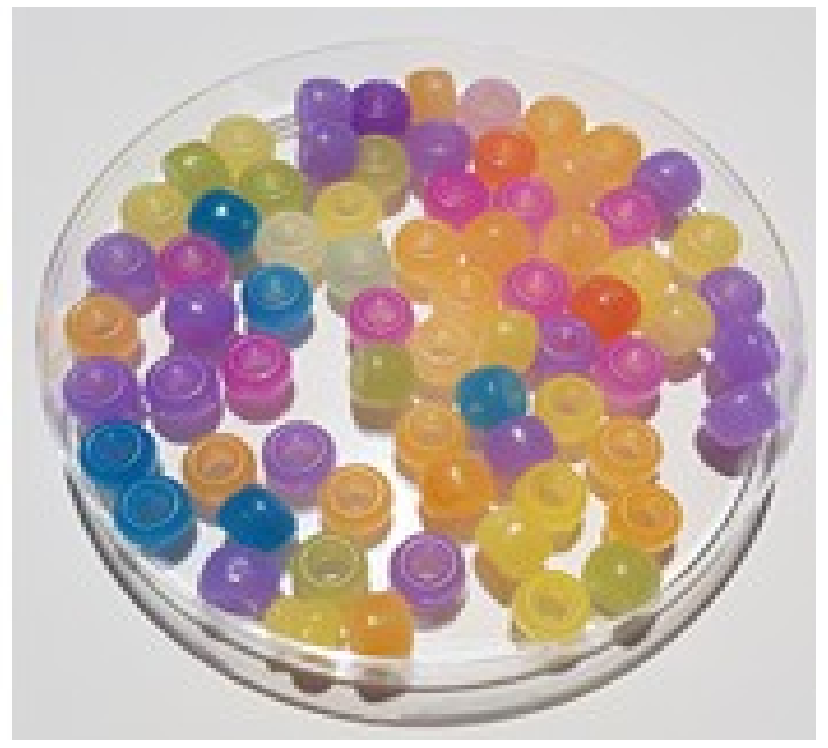

Figura 1. Las perlas UV contienen pigmentos fotocromáticos que se colorean en presencia de radiación UV.

\section{cevidente para muchos profesores. El procesamiento de la informacion y etana 2012), tan} evidente para muchos profesores. El procesamiento de la información y el aprendizaje interaccionan bioquímicamente en el cerebro con las emociones y, en consecuencia, el impacto de las emociones no se puede separar del aprendizaje y la racionalidad.

\section{Desarrollo de la propuesta}

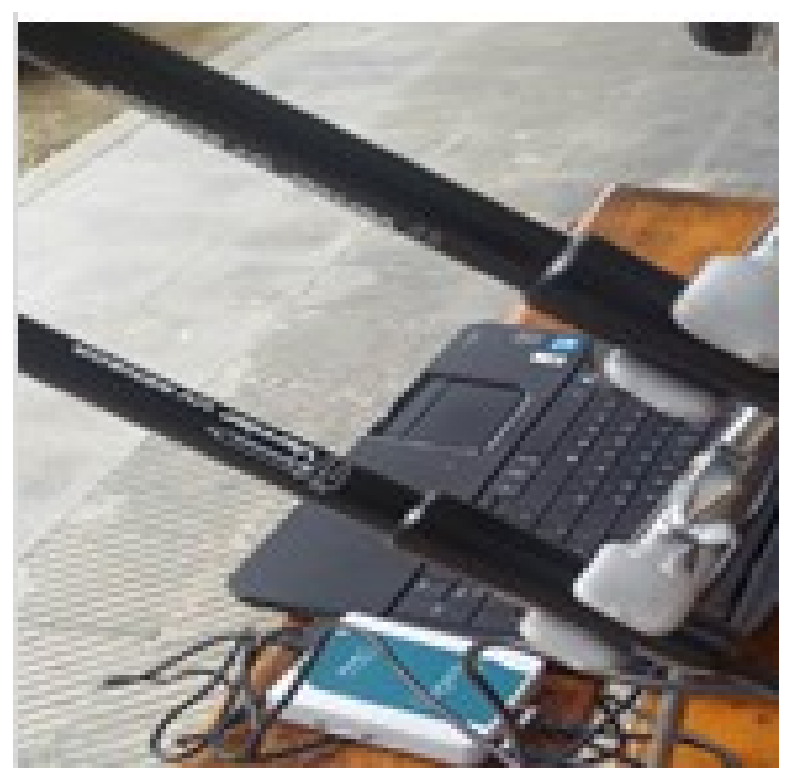

Figura 2. Los sensores de UV permiten medir la intensidad de radiación UVA y UVB.
- ¿Cuáles son los efectos de la radiación solar? (en diferentes materiales, en la piel, a nivel celular, en los ojos)

- ¿Cómo nos podemos proteger? (¿Qué contienen las cremas solares? ¿Qué son los fotoprotectores? ¿Qué tipos de protectores solares se pueden utilizar? ¿Qué otras medidas de protección hay?)

En nuestra experiencia, hemos podido comprobar que las preguntas generadas por los alumnos podían agruparse en torno a estos tres ejes con facilidad.

En las primeras actividades se busca despertar las emociones del alumnado (vivencias, sorpresa...) (figura 1). La neurofisiología ha puesto de manifiesto el fundamento emocional presente en todo sistema racional (García Retana 2012), tan

Todas las actividades que se presentan tienen elementos de indagación e investigación y se propone hacerlas en equipo. Tanto la realización de experimentos como el diseño experimental tienen una relevancia especial a lo largo de la propuesta, la finalidad es que los alumnos aprendan no solo ciencia sino sobre la ciencia. Las actividades experimentales son variadas, en muchos casos se utilizan materiales que son frecuentes en todos los centros. En algún caso de utilizan equipamientos algo más sofisticados, como por ejemplo sensores de radiación UV y espectrofotómetro de luz visible (figura 2).

En la tabla 1 se muestra una relación de las actividades que se proponen. 
Tabla 1. Relación de actividades que configuran la propuesta.

\begin{tabular}{|c|c|c|}
\hline Actividad & Tipología (Dificultad) & Objetivo \\
\hline ¿Y tú, te proteges del sol? & $\begin{array}{c}\text { Lápiz y papel y puesta en } \\
\text { común } \\
(+)\end{array}$ & $\begin{array}{c}\text { Detectar las ideas iniciales del alumnado e identificar } \\
\text { preguntas a las que queremos dar respuesta }\end{array}$ \\
\hline $\begin{array}{l}\text { Investigación del comportamiento de } \\
\text { las "perlas solares o fotocromáticas" } \\
\text { con diferentes tipos de luz. }\end{array}$ & $\begin{array}{l}\text { Trabajo experimental } \\
\qquad(++)\end{array}$ & $\begin{array}{c}\text { Diseñar experimentos con control de variables, recogida } \\
\text { de datos, análisis e interpretación de resultados }\end{array}$ \\
\hline $\begin{array}{c}\text { ¿Cómo es la luz solar? } \\
\text { Registro de espectros de diferentes } \\
\text { luces. }\end{array}$ & $\begin{array}{l}\text { Trabajo experimental } \\
\qquad(++)\end{array}$ & $\begin{array}{l}\text { Caracterizar diferentes tipos de radiación en la luz solar } \\
\text { utilizando un espectrofotómetro (SpectroVis) }\end{array}$ \\
\hline $\begin{array}{l}\text { ¿Cómo podemos medir la intensidad de } \\
\text { la radiación ultravioleta (UV)? } \\
\text { ¿Con qué unidades? ¿De qué depende? }\end{array}$ & $\begin{array}{l}\text { Trabajo experimental } \\
\qquad(++)\end{array}$ & $\begin{array}{l}\text { Identificar la intensidad cómo una característica de la } \\
\text { radiación y los factores de los que depende, utilizando } \\
\text { sensores UVA i UVB }\end{array}$ \\
\hline $\begin{array}{l}\text { ¿Cuál es la causa de los cambios } \\
\text { observados en las "perlas solares"? }\end{array}$ & $\begin{array}{l}\text { Lápiz y papel } \\
(++/+++)\end{array}$ & $\begin{array}{c}\text { Relacionar los cambios en las "perlas solares" con } \\
\text { cambios a nivel molecular }\end{array}$ \\
\hline $\begin{array}{c}\text { ¿Por qué los rayos UV pueden } \\
\text { constituir un riesgo para la salud? } \\
\text { ¿Por qué la alteración de las moléculas } \\
\text { de DNA producida por la radiación UV } \\
\text { constituye un riesgo para la salud } \\
\text { humana? }\end{array}$ & $\begin{array}{l}\text { Análisis y interpretación } \\
\text { de animación } \\
\qquad(+++)\end{array}$ & $\begin{array}{l}\text { Describir las fases del ciclo celular y los procesos que } \\
\text { tienen lugar en cada una de ellas. Identificar los agentes } \\
\text { reguladores. Relacionar las mutaciones con el } \\
\text { funcionamiento incorrecto del ciclo celular }\end{array}$ \\
\hline $\begin{array}{c}\text { ¿Cómo nos protege la capa de ozono y } \\
\text { la melanina? }\end{array}$ & $\begin{array}{l}\text { Análisis i interpretación } \\
\text { de imágenes i } \\
\text { animaciones } \\
(++)\end{array}$ & $\begin{array}{c}\text { Identificar los cambios químicos que ocurren en la capa } \\
\text { de ozono y relacionarlos con el efecto protector } \\
\text { Describir la estructura de la piel humana. Explicar la } \\
\text { función de los melanocitos i describir su situación en la } \\
\text { piel }\end{array}$ \\
\hline $\begin{array}{c}\text { Radiación solar y moléculas en la } \\
\text { atmósfera }\end{array}$ & $\begin{array}{l}\text { Análisis de animación } \\
\qquad(++)\end{array}$ & $\begin{array}{l}\text { Investigar los efectos de la radiación UV y otros tipos de } \\
\text { radiación (visible e IR) sobre moléculas de la atmósfera }\end{array}$ \\
\hline $\begin{array}{c}\text { ¿Por qué la radiación solar que nos llega } \\
\text { no es siempre igual? }\end{array}$ & $\begin{array}{l}\text { Lápiz y papel } \\
\qquad(++)\end{array}$ & $\begin{array}{l}\text { Interpretar el efecto de hora del día y la estación en la } \\
\text { radiación solar y la situación geográfica en la intensidad } \\
\text { de radiación solar que nos llega. } \\
\text { Identificar otros factores que influyen en la radiación } \\
\text { solar que nos llega }\end{array}$ \\
\hline Índice de radiación UV & $\begin{array}{l}\text { Papel y lápiz App móvil } \\
\qquad(+)\end{array}$ & $\begin{array}{c}\text { Relacionar el índice UV con la intensidad de radiación, y } \\
\text { los factores de que depende con su valor en diversos } \\
\text { lugares y distintas épocas del año }\end{array}$ \\
\hline ¿Qué es el melanoma? & $\begin{array}{l}\text { Interpretación de vídeos. } \\
\text { Uso de simulaciones de } \\
\text { laboratorio. } \\
(++)\end{array}$ & $\begin{array}{l}\text { Definir melanoma. Identificar los principales factores de } \\
\text { riesgo y principales señales de alarma en manchas en la } \\
\text { piel. Diferenciar al microscopio un tejido normal y uno } \\
\text { canceroso }\end{array}$ \\
\hline $\begin{array}{c}\text { ¿Por qué los humanos tenemos tantos } \\
\text { colores diferentes de piel? } \\
\text { Simulación de la acción de la selección } \\
\text { natural sobre el color de la piel } \\
\text { humana, }\end{array}$ & $\begin{array}{c}\text { Simulación con elementos } \\
\text { manipulables } \\
(+++)\end{array}$ & $\begin{array}{l}\text { Simular un proceso de adaptación por selección natural. } \\
\text { Evidenciar que la evolución es un proceso en el cual } \\
\text { intervienen el azar y la selección, y que una característica } \\
\text { favorable en un ambiente puede ser desfavorable en otro }\end{array}$ \\
\hline $\begin{array}{l}\text { Cremas solares comerciales. } \\
\text { ¿Qué información contienen las } \\
\text { etiquetas y los envases? }\end{array}$ & $\begin{array}{l}\text { Lápiz y papel } \\
\qquad(++)\end{array}$ & $\begin{array}{c}\text { Analizar y clasificar la información de etiquetas de } \\
\text { cremas solares comerciales e identificar los filtros solares } \\
\text { y el índice de protección. Interpretar el mecanismo de } \\
\text { acción de distintos tipos de filtros solares }\end{array}$ \\
\hline SPF (Factor de Protección Solar) & $\begin{array}{l}\text { Lápiz y papel } \\
\qquad(++)\end{array}$ & $\begin{array}{c}\text { Explicar el significado de SPF y utilizar las normativas } \\
\text { del etiquetado para argumentar su elección en situaciones } \\
\text { diversas } \\
\text { Relacionar el SPF la concentración de filtro solar }\end{array}$ \\
\hline
\end{tabular}


Tabla 1. Continuación

\begin{tabular}{|c|c|c|}
\hline Actividad & Tipología (Dificultad) & Objetivo \\
\hline $\begin{array}{l}\text { Investigar la eficacia de cremas solares } \\
\text { de distinto SPF utilizando sensores UV. }\end{array}$ & $\begin{array}{l}\text { Trabajo experimental } \\
\qquad(++)\end{array}$ & $\begin{array}{l}\text { Diseñar un experimento con control de variables, } \\
\text { recogida de datos, análisis e interpretación de resultados. } \\
\text { Comparar el \% de radiación retenida o reflejada por el } \\
\text { filtro solar en cremas de distintas marcas, precios, } \\
\text { caducidad... }\end{array}$ \\
\hline $\begin{array}{c}\text { ¿Por qué es necesaria la luz solar para la } \\
\text { salud? }\end{array}$ & $\begin{array}{l}\text { Papel y lápiz } \\
\qquad(+++)\end{array}$ & $\begin{array}{l}\text { Analizar e interpretar información sobre el origen y la } \\
\text { función de la vitamina D. }\end{array}$ \\
\hline $\begin{array}{l}\text { ¿Qué son y cómo actúan las cremas } \\
\text { solares de auto bronceado? }\end{array}$ & $\begin{array}{l}\text { Papel y lápiz } \\
\qquad(+++)\end{array}$ & $\begin{array}{c}\text { Analizar e interpretar información sobre la composición } \\
\text { y los efectos de cremas de auto bronceado. }\end{array}$ \\
\hline ¿Son peligrosas las camas solares? & $\begin{array}{l}\text { Papel y lápiz } \\
(++)\end{array}$ & Analizar datos y elaborar conclusiones \\
\hline $\begin{array}{c}\text { Investigar la eficacia de los cristales de } \\
\text { gafas de distinto filtro UV utilizando } \\
\text { sensores UV. }\end{array}$ & $\begin{array}{l}\text { Trabajo experimental } \\
\qquad(++)\end{array}$ & $\begin{array}{l}\text { Diseñar un experimento con control de variables, } \\
\text { recogida de datos, análisis e interpretación de resultados. } \\
\text { Comparar el \% de radiación retenida o reflejada por el } \\
\text { filtro solar de cristales de distinto filtro UV. }\end{array}$ \\
\hline ¿Como funciona el ojo? & $\begin{array}{l}\text { Trabajo experimental } \\
\qquad(++)\end{array}$ & $\begin{array}{l}\text { Simular en la una maqueta de ojo la formación de } \\
\text { imágenes en el ojo humano. Relacionar las } \\
\text { modificaciones de la curvatura del cristalino con la } \\
\text { acomodación. }\end{array}$ \\
\hline Disección de un ojo de buey o cordero & $\begin{array}{l}\text { Trabajo experimental } \\
\qquad(+++)\end{array}$ & $\begin{array}{c}\text { Identificar las partes de un ojo de mamífero. Comparar el } \\
\text { modelo fisiológico de ojo con la anatomía y fisiología del } \\
\text { ojo. }\end{array}$ \\
\hline $\begin{array}{l}\text { Debate: ¿Es saludable tomar el sol? } \\
\text { ¿Cómo? ¿Cuándo? ¿Cuánto? }\end{array}$ & $\begin{array}{l}\text { Juego de rol } \\
\qquad(++)\end{array}$ & $\begin{array}{l}\text { Argumentar a favor y en contra de las opiniones de } \\
\text { personajes utilizando los aprendizajes realizados durante } \\
\text { el proyecto, para tomar decisiones sobre actuaciones } \\
\text { saludables. }\end{array}$ \\
\hline
\end{tabular}

\section{Relación entre las ideas que se trabajan y los modelos científicos}

En didáctica de la ciencia parece estar clara la idea de que no se trata de enseñar una gran cantidad de conceptos inconexos, sino grandes ideas (Harlem 2010) también llamadas ideas clave (New Generation Science Standards 2013) o modelos teóricos (Izquierdo, Espinet, García, Pujol y Sanmartí 1999). En consecuencia, los conocimientos a aprender tendrán que ser pocos, pero claves y generales, útiles para comprender y explicar muchos fenómenos de manera que sean transferibles, es decir, aplicables a la interpretación de otros hechos y a la 
resolución de problemas no abordados específicamente en el marco del proyecto (Sanmartí y Márquez 2017).

En las propuestas ABP generalmente existe una cierta tensión entre el contexto escogido y las ideas científicas que el profesorado pretende que los estudiantes aprendan. Está tensión se puede resolver de dos formas: favoreciendo o las ideas del modelo teórico o las del contexto. En el primer caso, se trabajan el máximo de ideas del modelo, aunque no todas ellas sean necesarias para resolver, comprender o actuar en relación con la situación en la que se contextualiza el aprendizaje. En el segundo caso, sólo se plantea el aprendizaje de aquello que se necesita para resolver, comprender o actuar en relación con la situación en la que se contextualiza el aprendizaje. Generalmente, el objetivo no es tanto construir ideas clave o modelizar, sino instrumentalizar o aplicar conocimientos ya aprendidos y profundizar en competencias más transversales (Sanmartí y Márquez 2017). Posiblemente el futuro pasará por la combinación de los dos tipos de propuestas, la orientada a la construcción del modelo teórico y la orientada a su instrumentalización, a partir de combinar actividades que potencien las dos perspectivas (Domènech 2017).

Nuestra propuesta de proyecto intenta buscar un equilibrio entre contexto e ideas científicas. Se relaciona con modelos científicos relevantes: el modelo de célula y de ser vivo, el modelo de evolución por selección natural, el modelo energético de la luz, el modelo de cambio químico, el modelo materia o el modelo Sol-Tierra. Sin embargo, no se aborda el proceso de modelización completa de todos ellos, en cada una de las actividades se indican las relaciones entre las ideas que se trabajan y el modelo correspondiente, proporcionando así una nueva ocasión de contribuir a dicha modelización. Estamos considerando que modelizar no significa simplemente enunciar el modelo en cuestión, sino que significa ser capaz de construirlo, utilizarlo, de expresarlo para establecer relaciones y de evaluarlo.

Si se quiere profundizar más en un modelo determinado se podrían añadir aquellas actividades que se considere necesarias para abordar su construcción.

El proyecto incluye actividades para trabajar la composición y características de la radiación solar a partir de datos experimentales, como el registro de espectros de la luz visible y la medida de intensidad de radiación UVA y UVB. Las evidencias experimentales pueden utilizarse para la construcción de un modelo básico de luz, con el que los alumnos puedan explicar, comparar y predecir diferencias entre la luz visible y la UV en cuanto a su longitud de onda y contenido energético, así como diferenciar la energía de una determinada radiación y su intensidad. Los alumnos deben asociar el tipo de luz (visible, UVA, UVB) con una longitud de onda y un contenido energético.

La explicación de los efectos de la radiación UV requiere de la interacción materia-energía y el modelo cambio químico. Se propone explicar y predecir en base a estos modelos el efecto de la radiación solar en las perlas UV, en la capa de ozono, sobre el DNA y el mecanismo de acción de algunos protectores solares.

El análisis de los ingredientes de las cremas solares, así como la elaboración de cremas, están relacionados con el modelo materia, y su construcción, apropiación o utilización para que los alumnos puedan explicar y predecir las diferencias entre substancia y mezcla, y entre distintos tipos de mezcla, como las disoluciones y los sistemas dispersos, en especial las emulsiones. Se trabaja el concepto de solubilidad en relación con carácter hidrófobo o hidrófilo de los ingredientes de las cremas, y el de concentración en relación con la distinta proporción de filtro solar en las cremas elaboradas. La clasificación de los filtros solares en substancias orgánicas e inorgánicas permite establecer relaciones entre propiedades y estructura de las substancias, una de las ideas clave de la química. 
Se relaciona la respuesta del organismo humano a la radiación solar con la producción de melanina en los melanocitos presentes en la piel, es decir una respuesta que tiene lugar a nivel celular. De la misma manera, cuando se trabaja el efecto de cambios producidos a nivel molecular (en el ADN) en el control del ciclo celular y su relación con la aparición del cáncer, se está poniendo de manifiesto la relación entre actividad celular y actividad de organismo, es decir, de alguna manera se está contribuyendo a la construcción del modelo célula y del modelo ser vivo.

Así mismo, en una de las actividades, se relaciona la mayor cantidad de melanina con una menor alteración de ácido fólico por la radiación UV y, por tanto, con un mayor éxito reproductivo. Se estará utilizando, expresando y poniendo a prueba aquí el modelo de evolución por selección natural.

\section{Definición del producto final}

Una de les características inherentes a los proyectos es la presencia de un objetivo externo al aprendizaje que actúa como promotor de éste, es decir que justifica la necesidad de un aprendizaje en un contexto auténtico. Estamos hablando del producto final del proyecto. Este producto naturalmente debe relacionarse con el tema del proyecto, así como con las competencias que queremos que el alumnado desarrolle y es importante que esté definido desde el inicio, ya que será la guía para todo el proceso.

En nuestra propuesta se sugiere que el producto final sea una jornada abierta al resto de niveles del centro educativo donde los alumnos del curso en el que se ha desarrollado el proyecto organicen talleres, exposiciones y trípticos informativos utilizando el conocimiento construido sobre las características de los distintos tipos de protectores solares, la radiación UV y sus efectos en la salud y hábitos saludables. Los alumnos pueden elaborar pósteres para comunicar los resultados de sus investigaciones, sus aprendizajes y conclusiones. También pueden elaborar folletos informativos u organizar un evento tipo feria en la que los alumnos realicen algunos de los experimentos propuestos en las actividades, u otros relacionados. La situación ideal sería poder abrir estas muestras más allá de la propia aula o centro educativo, es decir, presentar el taller o la exposición a alumnos otros centros, a los padres, a entidades del barrio/municipio...

El producto final tiene que ser el resultado final de todo el proceso de indagación e investigación, tiene que servir para mostrar el recorrido que se ha hecho y proporcionar la oportunidad de comunicar lo que se ha aprendido.

La última de las actividades propuestas (tabla 1) es la realización de un debate en clase. La investigación en didáctica considera el uso de debates al aula de ciencias un buen contexto metodológico para favorecer el desarrollo del pensamiento crítico del alumnado y capacitarlo para aplicar el conocimiento y el pensamiento racional al análisis de problemas actuales. Esta capacitación le permitirá, en un futuro, poder participar en la sociedad como ciudadano activo y responsable. Es importante que el alumnado tenga claro que debaten para llegar a acuerdos, no es una competición dialéctica. Al final de la actividad tienen que recoger las propuestas consensuadas (Farró, Lope 2018). Alternativamente, podría proponerse esta actividad como producto final del proyecto.

\section{Evaluación del proyecto}

En cada actividad se indican los objetivos de aprendizaje y de ellos se desprenden los criterios de evaluación que, naturalmente, podrán ser variados en función de las modificaciones que cada profesor introduzca. Los indicadores de éxito resultaran a partir de las respuestas a preguntas como: "Cómo puedo evidenciar que el alumno es capaz de...?". 
En este sentido, el producto final que se propone (jornada de talleres, exposiciones de carteles, trípticos...) servirá para poner de manifiesto que han aprendido. Pero la evaluación no puede reducirse al producto final, será necesario prever momentos en los que el alumnado pueda reflexionar sobre los aprendizajes realizados y explicitarlos.

También será conveniente evaluar el trabajo en equipo. Sugerimos utilizar parrillas de observación que rellenará el profesor/a y utilizar también parrillas de autorregulación. De esta manera se obtiene información para el seguimiento individual de los miembros del grupo.

La propuesta que presentamos deja que el profesorado decida qué actividades del proyecto se utilizan para la regulación de los aprendizajes, para que tanto los alumnos como el profesorado conozcan el grado de consecución de los objetivos de aprendizaje, y poder intervenir en el caso que se detecte algún desajuste. El profesorado también puede utilizar algunas de las actividades del proyecto para evaluar individualmente el alumnado de manera que se contemplen los resultados en la calificación final. Los objetivos de aprendizaje y las demandas del proyecto han de ser compartidos con los alumnos, de la misma manera que los profesores han de decidir y compartir con el alumnado los criterios de evaluación.

Una propuesta de evaluación del aprendizaje de los alumnos, teniendo en cuenta que una finalidad importante del proyecto es incidir en los hábitos saludables del alumnado, es proporcionar a los alumnos la oportunidad de comparar sus ideas iniciales con las que tienen al final del proyecto. En este sentido, y al final del proyecto, el profesorado puede realizar, también para conocer el grado de adquisición o consecución de contenidos y destrezas, una actividad final para evidenciar si los alumnos han modificado su idea inicial respecto a protectores solares y si son capaces de argumentar sus afirmaciones utilizando conocimiento científico básico de los tres ejes del proyecto. Se realizó una actividad final de estas características para valorar los resultados de aprendizaje en la implementación piloto del proyecto con alumnos de $3^{\circ}$ ESO del instituto Jaume Balmes (Barcelona). Los resultados se exponen en el apartado de resultados de la implementación piloto.

Algunos profesores, que conocieron el proyecto en jornadas y talleres realizados, y que han utilizado actividades del proyecto con alumnos de $3^{\circ} \mathrm{ESO}$ durante las clases de ciencias, nos compartieron como realizaron la evaluación. En la evaluación se ha priorizado el producto final, y se ha llevado a cabo mediante coevaluación de los folletos o del póster sobre cremas solares elaborados por los alumnos. En algunas de las actividades, como la investigación del efecto de diversas cremas, se ha evaluado el informe individual realizado por cada alumno, y se ha utilizado para la calificación. La actividad del debate sobre el problema de los riesgos/beneficios del sol en el patio del centro escolar, que se propone como una de las actividades finales, ha sido también evaluada en base a la capacidad de argumentación de los alumnos utilizando el conocimiento científico desarrollado durante el proyecto.

El profesorado del centro que ha utilizado actividades del proyecto con todos los alumnos de $1^{\mathrm{o}}$ de bachillerato internacional ha tenido en cuenta para la evaluación y la calificación final del proyecto la carpeta de aprendizaje, la producción final, así como la participación y actitud del alumnado. La carpeta de aprendizaje incluye las actividades realizadas durante el proyecto con los datos recogidos en los experimentos, las conclusiones y la respuesta a las preguntas de cada actividad, así como un breve texto de reflexión individual de 50 palabras sobre los aprendizajes más destacados realizados durante el proyecto, ya sean respecto a la forma como se ha trabajado, o sobre los contenidos trabajados y las competencias desarrolladas. El producto final es un póster elaborado en grupo y presentado en un evento de centro para concienciar a los alumnos de los cursos de la ESO sobre la importancia de la protección solar, mientras que en el curso anterior fue una presentación oral acompañada de soporte visual 
realizada en clases de la ESO. Para la evaluación de cada uno de estos elementos se utilizaron rúbricas compartidas con los alumnos.

\section{Temporización}

La duración del proyecto es variable en función de los objetivos que cada centro priorice, las actividades que realice y la distribución entre horas dentro y fuera del centro. Si consideramos la realización de todas las actividades de la propuesta, más algunas actividades complementarias para contribuir a la construcción de modelos relacionados con el proyecto, pueden dedicarse alrededor de 30-35 h. Evidentemente el tiempo sería inferior si los alumnos utilizan (y no construyen) algunos de los modelos, que ya han sido construidos anteriormente. $\mathrm{Si}$ se focaliza en uno de los tres ejes del proyecto y se selecciona un número limitado de actividades relacionadas con este eje, la temporización puede adaptarse a 8-10 h de clase de ciencias de la naturaleza de $3^{\circ}$ ESO o de física y química o de biología y geología de $4^{\circ}$ ESO. También pueden incorporarse, con un objetivo específico, algunas actividades de la propuesta en unidades didácticas u otros proyectos.

A modo de ejemplo, en el centro en el que se han utilizado diversas actividades de la propuesta para elaborar un proyecto realizado por todos los alumnos de $1^{\circ}$ del bachillerato internacional, se han dedicado $14 \mathrm{~h}$ lectivas distribuidas de la siguiente manera: presentación del proyecto $\mathrm{y}$ actividades de indagación con las perlas UV y distintas lámparas UV (3 h), actividades relacionadas con los efectos biológicos de la radiación solar $(2 \mathrm{~h})$, actividades de investigación de la eficacia de distintas cremas solares, algunas de ellas elaboradas por los alumnos (6 h), y contribuciones a la carpeta de aprendizaje, elaboración de un póster para concienciar sobre el uso de protección solar para presentado en un evento escolar $(3 \mathrm{~h})$.

\section{Resultados de la actividad final de la implementación piloto}

Antes de completar la propuesta de actividades de proyecto y elaborar las versiones definitivas de las actividades, se realizó una implementación piloto $(4 \mathrm{~h})$ con 19 alumnos de $3^{\circ}$ ESO del instituto Jaume Balmes (Barcelona). Se implementaron ocho actividades que incluían las versiones iniciales de todas las actividades experimentales, dos actividades para trabajar los efectos de la UV, la actividad inicial de detección de ideas y compartición de objetivos del proyecto, y una actividad final diseñada para evidenciar si los alumnos evolucionaron en sus ideas iniciales sobre protectores solares, y en qué grado incorporaron conocimiento científico en sus argumentos sobre la importancia del uso de protectores solares utilizando los modelos y las ideas científicas trabajadas en los tres ejes del proyecto: 1) naturaleza de la radiación solar y diferencias de intensidad de radiación, 2) características de los tipos de fotoprotectores, composición y manera de actuar de los protectores solares, y 3) efectos sobre la salud (a nivel molecular, piel, ojos...).

Los resultados del análisis de la actividad final muestran un cambio importante respecto a la actividad inicial puesto que 16 de los 19 alumnos no estaban de acuerdo con la afirmación de que no hacía falta ponerse crema solar si se tenía la piel resistente y sólo dos alumnos no estaban de acuerdo en que era conveniente utilizar gafas de sol. Este resultado contrasta la actividad inicial que presentaba el mismo diálogo entre adolescentes, en la cual sólo 7 de los 19 alumnos no estaba de acuerdo con la afirmación de que no hacía falta ponerse crema solar, y una gran mayoría no estaba de acuerdo en que era conveniente utilizar gafas de sol.

En cuanto a los resultados de aprendizaje esperados, que los alumnos fueran capaces de utilizar de manera coherente y con el adecuado nivel de rigor argumentos basados en ciencia respecto a la importancia del uso de protectores solares, podemos afirmar que los alumnos elaboraron argumentos bien fundamentados en las ideas clave trabajadas en las actividades. 
Algunos alumnos utilizaron adecuadamente argumentos de más de uno de los tres ejes del proyecto, y es de destacar que tan solo dos alumnos no utilizó argumentos científicos para justificar el uso de las cremas solares.

Los argumentos de los alumnos, en la mayoría de los casos, estaban relacionados con la presencia de UVA y UVB en la radiación solar que llega a la Tierra (13/19) y con el contenido de las cremas solares y como éstas nos protegen del sol (13/19). Otros estaban relacionaban con los efectos a nivel molecular de la luz UV y los efectos sobre el organismo (8/19). Los argumentos se basaban en evidencias experimentales como los cambios de color en las perlas UV, los espectros registrados, y los valores de la intensidad UV con y sin crema solar, y otros en el contenido de las etiquetas de las cremas solares. La presencia de menor número de argumentos basados en los efectos producidos por la radiación UVA y UVB puede atribuirse a que se dedicó más tiempo a las actividades experimentales con las perlas UV y los sensores, y en la preparación y comparación entre cremas solares, que a las actividades en las que se proponía discutir sobre los efectos.

En conclusión, los resultados mostraron que los alumnos de $3^{\circ}$ ESO evolucionaron en su percepción de la importancia de utilizar convenientemente protectores solares, y construyeron argumentos coherentes relacionados con los tres ejes de contenido del proyecto.

\section{Reflexión sobre los resultados de la experimentación de la propuesta}

En cuanto a la implementación piloto de algunas actividades del proyecto, incluidas todas las experimentales, con un grupo de alumnos de $3^{\circ}$ de la ESO del instituto de secundaria INS Jaume Balmes (Barcelona) destaca que los alumnos trabajaron con bastante autonomía y se desenvolvieron bien en el uso de los sensores y en el diseño e interpretación de los resultados. También se puso de manifiesto que las preguntas a las que buscan respuesta los alumnos, y que se expresaron en el apartado punto de partida, pueden organizarse y encajarse con los tres ejes que constituyen el proyecto. En esta implementación se detectó la necesidad de incorporar actividades sobre los efectos beneficiosos del sol y su rol en la síntesis de vitamina $\mathrm{D}$, puesto no contemplarlos conduce una visión sesgada de los efectos de la luz solar, y a identificar sólo los posibles riesgos. Se detectó también la necesidad de incorporar actividades sobre los efectos de la radiación de las camas solares, debido a las numerosas dudas que plantearon los alumnos. Los resultados de la implementación se incorporaron en la versión final de las actividades y se añadieron estas nuevas actividades. Tal y como se ha comentado en el apartado de evaluación del proyecto, los resultados de la actividad final de la implementación se muestran en el apartado dedicado a la implementación piloto.

El profesorado de La Salle Bonanova (Barcelona) que utilizó la versión final de las actividades del proyecto con todos sus alumnos de $1^{\circ}$ de bachillerato internacional lo valoraron como un proyecto con gran aceptación y aprendizajes por parte de los alumnos.

El proyecto, y en especial sus actividades experimentales, han sido presentados en diferentes jornadas de formación de profesorado, y en algunas de ellas participaron también alumnos: IV Jornada CTM, abril 2017, Universidad de Andorra, STEAMConf Barcelona, abril 2017, III Conferencia Internacional de Educación, abril de 2017, y EQUÍPA’T de bones pràctiques a secundària, julio 2017, ICE de la Universitat de Girona. El feedback del profesorado ha sido siempre muy positivo, y los alumnos se implicaron en la realización de las actividades y trabajaron con aprovecho, motivación e interés. Las actividades del proyecto, así como las orientaciones didácticas pueden ser consultadas y descargadas en la aplicación ARC (Aplicació de Recursos al Currículo) del Departament d'Ensenyament en: http://apliense.xtec.cat/arc/node/30700. 


\section{Referencias}

Domènech J. (2017) Treball per projectes i competència científica: una proposta metodològica per al disseny de Projectes d'Indagació. Perspectiva Escolar.

Farró L., Lope S. (2018). Cartas conversacionales para aprender a debatir. Alambique: Didáctica de las ciencias experimentales, (91), 49-55.

Fortus D., Krajcikb J., Dershimerb R. C., Marx R. W., Mamlok-Naamand R. (2005) Designbased science and real-world problem solving. International Journal of Science Education 27 (7) $855-879$.

García Retana J. Á. (2012) La educación emocional, su importancia en el proceso de aprendizaje. Educación 36(1), 2-13.

Harlen W. (2010) Principios y grandes ideas de la educación en ciencias. Gran Bretaña. Publicado por Association for Science Education.

Hazelkorn Ellen, Ryan Charly, Beernaert Yves, Constantinou Constantinos , Ligia Deca , Grangeat Michel , Karikorpi Mervi, Lazoudis Angelos , Pinto-Casulleras Roser , Manuela Welzel-Breuer . Science Education for Responsible Citizenship. EUR 26893. 2015; Brussels: European Commission - Research and Innovation.

Izquierdo M., Espinet M., García M. P., Pujol R. M., Sanmartí N. (1999) Caracterización y fundamentación de la ciencia escolar. Enseñanza de las Ciencias. Vol. No extra, 79-91.

New Generation Science Standards (2013) Recuperado de: http://www.nextgen science.org/

Sanmartí N., Márquez C. (2017) Aprendizaje de las ciencias basado en proyectos: del contexto a la acción. Ápice. Revista de Educación Científica 1(1), 3-16. DOI: https://doi.org/10.17979/arec.2017.1.1.2020

Wang H. H., Moore T. J., Roehrig G. H., Park M. S. (2011) STEM integration: Teacher perceptions and practice. Journal of Pre-College Engineering Education Research 1 (2), 2. 